\title{
Adjustment to the psychological and social sequelae of recurrent genital herpes simplex infection
}

\author{
John L Brookes, Serena Haywood, John Green
}

\begin{abstract}
Objective-To investigate whether adverse psychological consequences and impaired sexual and interpersonal functioning are present in individuals suffering from recurrent genital herpes simplex infection (HSV)

Design-A questionnaire-based study completed by subjects defined as having more than one episode of HSV infection.

Subjects-Completed questionnaires were returned from 90 subjects; 40 from the department of genitourinary medicine at our hospital and 50 from members of the Herpes Association.

Results-Stress and being physically run-down were identified as provoking factors by the majority of individuals (78\% and $56 \%$ respectively). The frequency of sexual activity was not different when comparing rates before and after infection ( $<<0.001)$ and the majority of subjects had told partners of their infection. Women reported significantly greater disturbances in several psychological variables and reported a greater decrement in their general health. However, overall there were no differences in the psychological sequelae following herpes infection.

Conclusions-The study suggests that, given time, most people are able to adjust psychologically to having recurrent herpes infection. However, for a minority of subjects this is not the case and these individuals may require psychotherapeutic intervention.
\end{abstract}

(Genitourin Med 1993;69:384-387)

\section{Introduction}

A number of published studies have looked at the impact of genital herpes infection on psychological well-being and on sexual functioning in people with herpes infection. Most of these studies have been either of acute cases in the early stages after infection or of patients being referred for psychotherapy or counselling. ${ }^{12}$ These studies report that people with herpes have many psychological problems as a result of their infection and have problems with sexual functioning. What the studies are unable to show is what happens to these patients over time. Do they remain disturbed or, over time, do they come to terms with their infection?

Published studies have usually not used a control group. It is unclear from many of them whether patients with herpes tend to be more psychologically distressed as a result of having herpes or whether they might have been distressed prior to contracting the infection. There is clear value in getting individuals to assess these areas of their lives relative to their premorbid levels. While this relies on the memory of patients it allows them to act as their own retrospective controls, and gives some indication of how well individuals themselves feel that they have adjusted to having herpes.

The current literature on psychological variables in genital herpes infection also fails to identify what links, if any, there are between these factors and sexual functioning. This is clearly important to establish.

We therefore aimed to assess whether adverse psychological consequences and impaired sexual and interpersonal functioning as a result of genital herpes infection are present in individuals who have had the infection over a lengthy period of time. We also aimed to look at what patients themselves perceived as the provoking factors for recurrent attacks. An area of particular interest was their perceptions of the role played by stress in provoking attacks since the widespread belief that stress leads to reactivation has not been substantiated in any major study. ${ }^{3-5}$

\section{Patients and methods}

The study sample was drawn from two sources. Forty patients attending a GUM clinic for advice on recurrent herpes simplex virus infection (HSV) were approached via a research nurse to participate in the study. There were no refusals. A further 50 subjects were recruited via the Herpes Association through a postal questionnaire sent to a randomly selected sample of individuals selected from the organisation's membership list. The response rate was $60 \%$. The sample contained 40 men and 50 women.

Subjects were asked to complete a 55 item questionnaire. The first 25 questions covered demographic details, religious beliefs, relationships, disease pattern and symptoms, provoking factors, premonitory symptoms, treatment including self-treatment and sexual behaviour.

The severity of symptoms experienced were rated on a seven point scale $(1=$ most severe, $7=$ least severe). These symptoms were genital ulceration, pain or swelling in the groin, feeling generally unwell, discomfort 
of the genitals, painful urination and bleeding/discharge. An overall symptom score was calculated by adding these scores together.

To assess the effect of HSV infection on sexual activity, subjects were asked how often they had had sex in the six months prior to infection, in the first six months after infection and in the past six months. For the two post-herpes questions subjects were asked to separate sex during attacks from sex between attacks. Subjects were also asked whether herpes infection was the main cause for any change in sexual activity. Statistical analysis was performed using the Wilcoxon matched pairs test.

The remaining 30 items were a series of statements about the subject's attitudes to sexual performance and enjoyment, work, relationships with partners, family and friends, self-image and attitudes to the infection. Subjects were asked to rate whether these statements applied to them more or less than before they contracted herpes on a series of seven-point scales, a score of 4 representing no change. These items were derived from other published studies in the literature ${ }^{14}$ which have looked at psychological variables in herpes. Since most of these studies were reporting on these factors in those early in the course of their herpes infection this allows examination of the extent to which psychological disturbance resolves over time in people with herpes.

\section{Results}

Respondents had a median age of 34 years (range 19-60) with a median duration of infection of 6 years (range 1-29). All subjects had had at least two recurrences of herpes after the primary attack. Sixty six subjects had had more than 10 recurrences $(73 \%)$. The majority of subjects were from social classes 1 and 2 (70 subjects; $78 \%$ ) with 61 subjects $(68 \%)$ being educated to at least " $A$ " level.

Overall, $57(63 \%)$ individuals were single, $19(21 \%)$ were married and the remainder divorced or separated. Eighty three subjects $(92 \%)$ identified themselves as heterosexual, five men $(5 \%)$ identified themselves as gay and two women (2\%) as bisexual. For heterosexuals the median number of sexual partners in the past 3 years was six for men (range $0-40$ ) and three for women (range 0-25). For homosexual men, the median number of partners was 35 (range 2-100).

Median age at first intercourse was 18 years (range 13-30 years). This was quite a highly sexually active population, which was reflected in their lifetime rate of other sexually transmitted diseases, with 42 subjects $(47 \%)$ of the whole sample having had at least one other STD.

Forty four subjects (49\%) were in a sexual relationship which had lasted at least 6 months, $10(11 \%)$ in a relationship which had lasted 2-6 months and $35(39 \%)$ were not currently in a sexual relationship.

Time since first infection was not correlated with overall symptom severity or with severity of any of the individual symptoms. There was no relationship between symptom severity and number of sexual partners, sex of the subject or whether they were in a relationship currently. Most of our subjects reported using painkillers but this was not related to overall severity of attacks or, interestingly, the amount of pain they reported having. For women, the more symptoms they had, the more likely they were to have difficulty in obtaining an orgasm ( $\mathrm{r}=0.38, \mathrm{p}<0.001)$. There was no correlation for men.

\section{SYMPTOM SEVERTTY}

The mean overall symptom severity was 20 (range 3-43) so that this was a group of subjects with relatively severe symptoms. The symptoms which were felt to be most troublesome by subjects were pain (mean score $2 \cdot 6$ ), feeling unwell (mean score $2 \cdot 6$ ) and painful urination (mean score $2 \cdot 7$ ).

\section{Provoking factors}

Table 1 shows the percentage of subjects identifying particular factors as provoking attacks. It can be seen that stress, other causes of ill health and menstruation were the most commonly identified factors. These are of course the beliefs of patients about what provokes their attacks.

\section{Effects on sexual activity}

Subjects reported no differences between current frequency of sexual activity and frequency of sexual activity prior to the first attack of herpes. However they reported that the frequency of sexual activity during the first six months after infection was reduced both during and between attacks (Wilcoxon matched pairs $p<0.0001$ and $p<0.001$ respectively). Current activity during an attack was reduced relative to periods between attacks and to levels of sexual activity prior to infection (Wilcoxon matched pairs $\mathrm{p}<0.0001$ ). A number of subjects reported that they did have sexual activity during attacks.

Table 2 shows the percentage of patients who have told, who have not told and who do not know whether they will tell past and future partners about their infection.

Table 1 Percentage of subjects identifying factors as provoking attacks

\begin{tabular}{lr}
\hline Stress & 78 \\
Being physically run-down & 56 \\
Sexual intercourse & 38 \\
Menstruation (women only) & 50 \\
Other physical illness & 24 \\
Ultraviolet light & 12 \\
Jetlag & 10 \\
Alcohol & 6 \\
Weather & 5 \\
Steroids & 2 \\
Other medications & 2 \\
Opiate drugs & 1
\end{tabular}

Table 2 Percentage of subjects who would or would not tell past or future partners of their infection

\begin{tabular}{lll}
\hline & Past Partners & Future Partners \\
\hline Have told & 60 & 68 \\
Have not told & 30 & 11 \\
Don't know or undecided & 10 & 21 \\
\hline
\end{tabular}




\section{Psychological variables}

Table 3 shows the ratings of individuals on psychological variables. It can be seen that on none of the questions did subjects report average scores of one standard deviation or greater away from the midpoint. Thus they were essentially reporting, on average, that they had not changed on the questions asked relative to before they got herpes. Adding scores on all the psychological variables together yielded a mean of 78 with a standard deviation of 16.9 , as opposed to an expected score if no-one reported any change of $\mathbf{8 4}$. Thus there was no overall indication of change since acquiring herpes.

However, examining the data in more detail, there were significant differences between the sexes in their answers to some of the questions. Women reported significantly greater reductions in how desirable they felt sexually $(t=2 \cdot 25, p<0.05)$, in how confident they felt $(t=3.61, p<0.001)$, felt more dirty $(t=2.37, p<0.05)$ and reported a greater decrement in their general health $(t=2.87, p<0.01)$ since getting herpes. They also reported that they were more likely to worry about future attacks $(t=2.04$, $\mathrm{p}<0.05)$. Women were also less likely to report being angry with the person who had given them herpes than men $(t=2.04$, $\mathrm{p}<0.05$ ).

Individuals who reported that they were currently in a sexual relationship reported increased levels of interest in sex relative to before they contracted herpes $(t=-1.99$, $p<0.05)$ and greater enjoyment of sex $(\mathrm{t}=-3.48, \mathrm{p}<0.001)$ but no differences were seen with respect to ease of getting an orgasm, fear of sex or difficulties in getting aroused.

The number of sexual partners an individual had had over the past three years was unrelated to any of the questions on sexual functioning except difficulty in becoming aroused where there was a small but significant correlation $(r=0.32, p<0.01)$.

In the sample as a whole $32 \%$ of subjects reported that they had increased the frequency with which they had had sex since getting herpes and $22 \%$ had reduced it. Five

Table 3 Mean and standard deviation of psychological variables rated from 1 (much less than before contracting herpes) to 7 (much more than before)

\begin{tabular}{|c|c|c|}
\hline & Mean Rating & S.D. \\
\hline $\begin{array}{l}\text { Capable of warmth and intimacy } \\
\text { Sexual desirability } \\
\text { Cutting oneself from everybody } \\
\text { Relationship with family } \\
\text { Relationship with friends } \\
\text { Relationship with partner } \\
\text { Feeling sad } \\
\text { Feeling guilty } \\
\text { Suicidal feelings } \\
\text { Worry more than before about health } \\
\text { Confidence } \\
\text { Feeling dirty } \\
\text { Feeling punished for sexual activity } \\
\text { General health worse } \\
\text { Interest in sex } \\
\text { Enjoyment of sex } \\
\text { Ability to achieve orgasm } \\
\text { Fear of sexual intercourse } \\
\text { Ability to become aroused } \\
\text { Sexual attractiveness }\end{array}$ & $\begin{array}{l}3 \cdot 8 \\
3 \cdot 2 \\
3 \cdot 7 \\
4 \cdot 1 \\
3 \cdot 9 \\
4 \cdot 0 \\
3 \cdot 4 \\
3 \cdot 5 \\
3 \cdot 8 \\
3 \cdot 6 \\
3 \cdot 5 \\
3 \cdot 7 \\
3 \cdot 6 \\
3 \cdot 5 \\
3 \cdot 5 \\
3 \cdot 7 \\
4 \cdot 0 \\
3 \cdot 8 \\
4 \cdot 3 \\
3 \cdot 8\end{array}$ & $\begin{array}{l}1.42 \\
1.38 \\
1.36 \\
0.99 \\
0.66 \\
1.42 \\
1.68 \\
1.40 \\
1.48 \\
1.25 \\
1.40 \\
1.42 \\
1.49 \\
1.40 \\
1.11 \\
1.26 \\
0.95 \\
1.46 \\
1.00 \\
1.52\end{array}$ \\
\hline
\end{tabular}

individuals had not had a sexual partner in the past three years.

Comparing those subjects who reported that the frequency with which they had sex had stayed the same or had increased since they contracted herpes against those who reported that their frequency of intercourse had declined, there were some differences on psychological variables. Individuals whose frequency of sex had reduced reported a reduced capacity for warmth and intimacy $(t=3.42, p<0.001)$, a reduced interest in sex $(t=3.25, p<0.01)$, a reduced ability to have an orgasm $(t=2.29, p<0.05)$, a reduced enjoyment of sex $(t=3.78$, $\mathrm{p}<0.001)$. They also reported better relations with family $(t=-1.98, p<0.05)$, but worse with friends $(t=2.30, p<0.05)$ and their partner $(t=3.27, p<0.01)$.

\section{Discussion}

The study population were individuals who were, for the most part, chronic herpes sufferers, who had had the disease for an average of six years. Unlike some other studies such as that reported by Drob et al individuals had not sought assistance with psychological aspects of their herpes. In this present study, however, we have clearly investigated subjects from two highly selected populations who may differ in terms of their psychological adjustment to herpes infection. It may well be that the subjects recruited from our GU clinic are more distressed and more psychologically disturbed than the subjects recruited from the Herpes Association who may have more emotional support and hence may adjust more fully and less damaging than patients with lower levels of social backup. Also, in attempting to quantify psychological factors before and after our subjects developed herpes we are using the subjects as their own control and we acknowledge the limitations that such a comparison may have.

The most interesting feature of the data, however, is that most subjects reported that they were essentially functioning at the same level as they had been prior to contracting herpes. They were as sexually active as they had been, they enjoyed sex and functioned well in sex. There was no evidence that, for most subjects, having herpes had influenced the way in which they saw themselves, their ability to relate to others in intimate situations or their feelings of attractiveness. This contrasts with other studies using more acute populations where reports of psychological distress are common. ${ }^{12}$ The most sensible interpretation of this is simply that, given time, most people come to terms with having herpes and are able to function well sexually and generally. This is also consistent with clinical experience and more recent papers, as opposed to the earlier studies when genital herpes was regarded both by the medical profession and by the media and public as a much more stigmatised disease.

Half the subjects were in a long-term relationship so that having herpes clearly did not 
prevent them from getting or retaining a partner. We do not know, of course, what proportion of long-term partners themselves had herpes.

In contrast to this generally favourable picture, however, there were some specific problems for the women in the sample. While they functioned sexually as well as they had before, the attitude of some women towards themselves did seem to have worsened chronically as a result of having herpes in a way that had not occurred in men. Women were more likely to feel dirty, less desirable sexually and less confident generally after getting herpes. They were also more likely to have concerns about their general health and to worry about getting future attacks. It may be that particular attention needs to be paid to women with chronic herpes attacks by genitourinary medicine clinics.

A proportion of subjects appeared to have continuing problems, even after years of having herpes. They reported negative feelings about themselves and these were reflected in a lower level of sexual activity. It is easy to assume that negative feelings about oneself and reduced sexual activity are natural consequences of having herpes. However, the data over all showed that this is not so. Patients who show continuing reduced sexual activity and functioning and continuing problems with their feelings about themselves probably need to be carefully reviewed with a view to possible counselling or referral on for support or therapy.

The issue of informing partners did lead to some concern. Clearly most subjects had told their partners and expected to tell their future partners, but there were significant numbers of individuals who either had not told past partners or were unwilling to say if they had or not. More worryingly, about a quarter of the sample said either that they would not tell future partners or would not commit themselves. More emphasis may need to be given to helping patients to get into a position where they feel able to inform future partners of their infection.

It is easy to assume with any illness that patients' minds are blank tablets on which the physician writes the necessary information about their illness unhindered by the preconceptions of the patient or the interference of other sources of advice. It is clear that for chronic herpes patients this is not the case. They have clear ideas, whether accurate of not, about the things that they think provoke attacks and it is important to be aware of these when advising patients about the management of their infection. Similarly patients are likely to have a whole range of sources of advice. There is good evidence that, for most health issues, it is easier to fill in gaps in knowledge than to correct misconceptions. There may be an advantage in trying to establish with patients with chronic herpes what their own understanding of the disease is and not simply to assume that it is the same as the physician's.

Overall, then, the study suggests that, given time, most people are able to adjust psychologically to having chronic herpes attacks. They are also able to enjoy a satisfactory sex life in spite of their infection. There would appear nevertheless to be a minority of subjects for whom this is not the case and it is important not to see their failure to adjust as being a natural result of having herpes and, hence, fail to intervene. ${ }^{7}$

We acknowledge the help and encouragement we received from Sarah Weston (Herpes Research Nurse), Dr David Goldmeier and Dr S Panja. This work was assisted by a financial award from the Wellcome Foundation.

1 Drob S, Loemer M, Lifshutz $H$. Genital herpes: The Psychological Consequences. Br 7 Med Psychol 1985;58: 307-15.

2 Goldmeier D, Johnson A, Byrne $M$, Barton $S$. Psychosocial implications of recurrent genital herpes
simplex virus infection. Genitourin Med 1988;64:327-30.

3 VanderPlate C, Aral SO, Magder L. The relationship among genital herpes simplex virus infection, Stress and Social Support. Health Psychol 1988;7:159-68.

4 Levenson JL, Hammer RM, Myers T, Hart RP, Kaplowitz LG. Psychological factors predict symptoms of severe recurrent genital herpes infection. $\mathcal{f}$ Psychosom Res 1987;31:153-9.

5 Kemeny ME, Cohen F, Zegans LS, Conant MA. Psychological and immunological predictors of genital herpes recurrence. Psychosom Med 1989;51:195-208.

Zigmund AS, Snaith PR. The Hospital Anxiety and Depression Scale. Acta Psychiatr Scand 1983;67:361-70.

7 Rose FB, Camp CJ. Genital herpes: how to relieve patient's physical and psychological symptoms. Postgrad Med 1988;84:81-6. 